\title{
New Insights on the Self-Assembly of Bio-sourced Block Copolymer MH-b-PS in Aqueous Solutions: Nanocorals, Cubosomes \& Nanocubes
}

\author{
Yomen Atassi*1,2 and Redouane Borsali ${ }^{1}$ \\ ${ }^{1}$ Univ. Grenoble Alpes, CNRS, CERMAV, 38000 Grenoble, France \\ ${ }^{2}$ Higher Institute for Applied Sciences and Technology, Damascus, Syria
}

* Corresponding Author's email: yomen.atassi@hiast.edu.sy

\section{Introduction:}

It is well known that the morphology or shape of nanomaterials influences a wide spectrum of their physical, chemical and even in-vivo biological properties [1]. The research for novel morphologies is still a trendy approach in the nanofabrication by the self-assembly [2]. For example, new mesoporous morphologies with high specific surface hold great promise for hetero-catalysis applications [3] or for bioengineering and separation applications [4]. Moreover, it is also well accepted that for applications such as drug delivery systems and drug release systems, nanoparticles have to fulfill strict dimensional criteria and should exhibit low polydispersity index [5]. On the other hand, cubosomes, which are nanostructured liquid crystalline particles, are usually made of certain amphiphilic lipids in definite proportions. They are remarkable drug delivery potential owing to their multicompartmental structure, thermodynamic stability, bioadhesion, ability of encapsulating hydrophilic, hydrophobic and amphiphilic substances and potential for controlled release through functionalization [6]. Recently, He et al have reported for the first time the preparation of cubosomes from hierarchical self-assembly of poly(ionic liquid) block copolymers: poly(acrylic acid)-block-poly(4-vinylbenzyl)-3-butyl imidazoliumbis(trifluoromethylsulfonyl)imide. They showed that the presence of the liquid ionic moieties is a prerequisite for cubosomes formation [7].

Furthermore, it has been proved that cubic polymeric microparticles show enhanced uptake by breast cancer cells because they offer a larger contact area with a cell compared to a sphere [8]. Two approaches are usually adopted to prepare those non-traditional morphologies. The first is the top-down approach, such as particle replication in nonwetting template (PRINT), lithography, and template induced printing [9]. The second one is the bottom-up approach by the self-assembly technique [10]. For the preparation of cubic polymeric nanoparticles self-assembly, two different methodologies have been adopted: Zhang et al. have proved that cubes with edge sizes between 200 and $600 \mathrm{~nm}$ can be formed by evaporation induced aggregation of polystyrene-blockpoly(acrylic acid) micelles in the presence of organic solvent at $70-120^{\circ} \mathrm{C}$ [11]. Another study conducted by $\mathrm{Tu}$ et al. showed that poly( $\varepsilon$-caprolactone) can form nanocubes upon evaporation 
from dichloromethane solution at $100^{\circ} \mathrm{C}$ [12]. Recently, Margulis et al. reported a simple method to form polymeric cubic nanoparticles by the aqueous self-assembly of amphiphilic dithiolanecontaining ABA triblock copolymers followed by evaporation-induced crystallization of the poly(ethyleneglycol) (PEG) central blocks $[13,14]$.

It is worthy to note that both cubosomes and nanocubes morphologies are mostly metastable due to thermodynamic favorability towards sphere nanoparticles formation. This implies that their formation is under kinetic control, and hence a laborious tuning of the experimental parameters is needed to forward the self-assembly nanofabrication towards cubosomes and nanocubes formation [10].

An interesting methodology for the generation of polymeric self-assembled nanoparticles is called nanoprecipitation. It uses the Ouzo effect. It was first developed by Fessi et al. [15], it is based on the partial miscibility of a polymer between two different solvents, where nanoparticles are precipitated from polymer chains in solution as a result of displacing a solvent by an anti-solvent [16]. The resultant mesophasic structure is sensitive to small variations of the assembling conditions including polymer composition and concentration, temperature, the nature and content of the common solvent and the anti-solvent $[16,17]$. Furthermore, mesophasic structures generated under kinetic control are also dependent on structure ageing and drying process [10].

In this work, the self-assembly of $\mathrm{MH}_{1.2 \mathrm{k}}-\mathrm{b}-\mathrm{PS}_{2.3 \mathrm{k}}$ in aqueous medium using the nanoprecipitation method has been revisited. The different experimental parameters have been tuned in order to tailor the generation of a myriad of novel self-assembly's morphologies ranging from nanocoral particles, through cubosomes to nanocubes. Nanocoral particles are novel mesoporous morphologies formed by nano-spheres with coral-like porous hollow architecture. They could have catalytic potentialities owing to their high specific area or they could be efficient for biosensing and bioengineering applications [3,4]. Cubosomes/hexosomes are simply formed by adjusting the concentration of the self-assembled copolymer in the anti-solvent. There is no need to the presence of an ionic liquid component in the copolymer structure. Moreover, the preparation of nanocubes based on this co-polymer has been investigated and the preparation is also validated on a higher molecular weight of the co-polymer MH1.2k-b-PS4.8k. To the best of the authors's knowledge, this is the first time those nanocubes has been generated using this type of bio-sourced co-polymer in aqueous solution and at room temperature.

\section{Materials and methods: Self-assemblies' procedures:}

First, $5.0 \mathrm{mg}$ of $\mathrm{MH} 1.2 \mathrm{k}-\mathrm{b}-\mathrm{PS} 2.3 \mathrm{k}$ was dissolved in $5.0 \mathrm{~g}$ of mixed solvents [4:1 (w/w) Acetone/water] and stirred overnight. Before use, polymer solution is sonicated for $10 \mathrm{~min}$ at $30^{\circ} \mathrm{C}$ under at $24 \mathrm{kHz}$ and $10 \mathrm{~W}$ (amplitude adjustment at 100\% and sonicator operated in the continuous mode).

\section{Spherical Nanoparticles Preparation}

The nanoparticles were prepared by following two different methods. Method A (standard nanoprecipitation): $2.5 \mathrm{~g}$ of the copolymer solution was slowly added dropwise to $100 \mathrm{~g}$ of Milli$\mathrm{Q}$ water using a Pasteur pipet during a customized period of time (50 s to get monomodal spherical nanoparticles) under vigorous stirring, with a stirring rate of $500 \mathrm{rpm}$. Method B (reverse nanoprecipitation nanoprecipitation): $100 \mathrm{~g}$ of Milli-Q water was slowly added to $2.5 \mathrm{~g}$ of the copolymer solution using a Pasteur pipet for $10 \mathrm{~min}$ under vortex stirring (stirring rate of $500 \mathrm{rpm}$ ). Subsequently, the suspensions obtained from both methods A and B were stirred vigorously for 2 $\mathrm{h}$ and acetone was removed by evaporation under reduced pressure at room temperature. 


\section{Nano-Coral Particles Preparation}

The same procedure as for spherical nanoparticles preparation, but the copolymer solution is added to water during $10 \mathrm{~min}$.

\section{Cubosomes Preparation}

The same procedure as for spherical nanoparticles preparation, but the suspension obtained from method A was stirred for $2 \mathrm{~h}$ and was concentrated to $4 \mathrm{~g}$ by evaporation under reduced pressure at room temperature.

\section{Nano-Cubes Preparation}

The same procedure as for spherical nanoparticles preparation. The aqueous micellar solution of the copolymer is deposited on a surface and dried slowly for at least $24 \mathrm{~h}$ at room temperature.

\section{Results and discussion:}

\section{1- Nanocral-like porous self-assembly}

Figure (1) shows the micrographs of nanocoral-like porous morphology that could have potential applications in heterogeneous catalysis and bioengineering applications, etc.

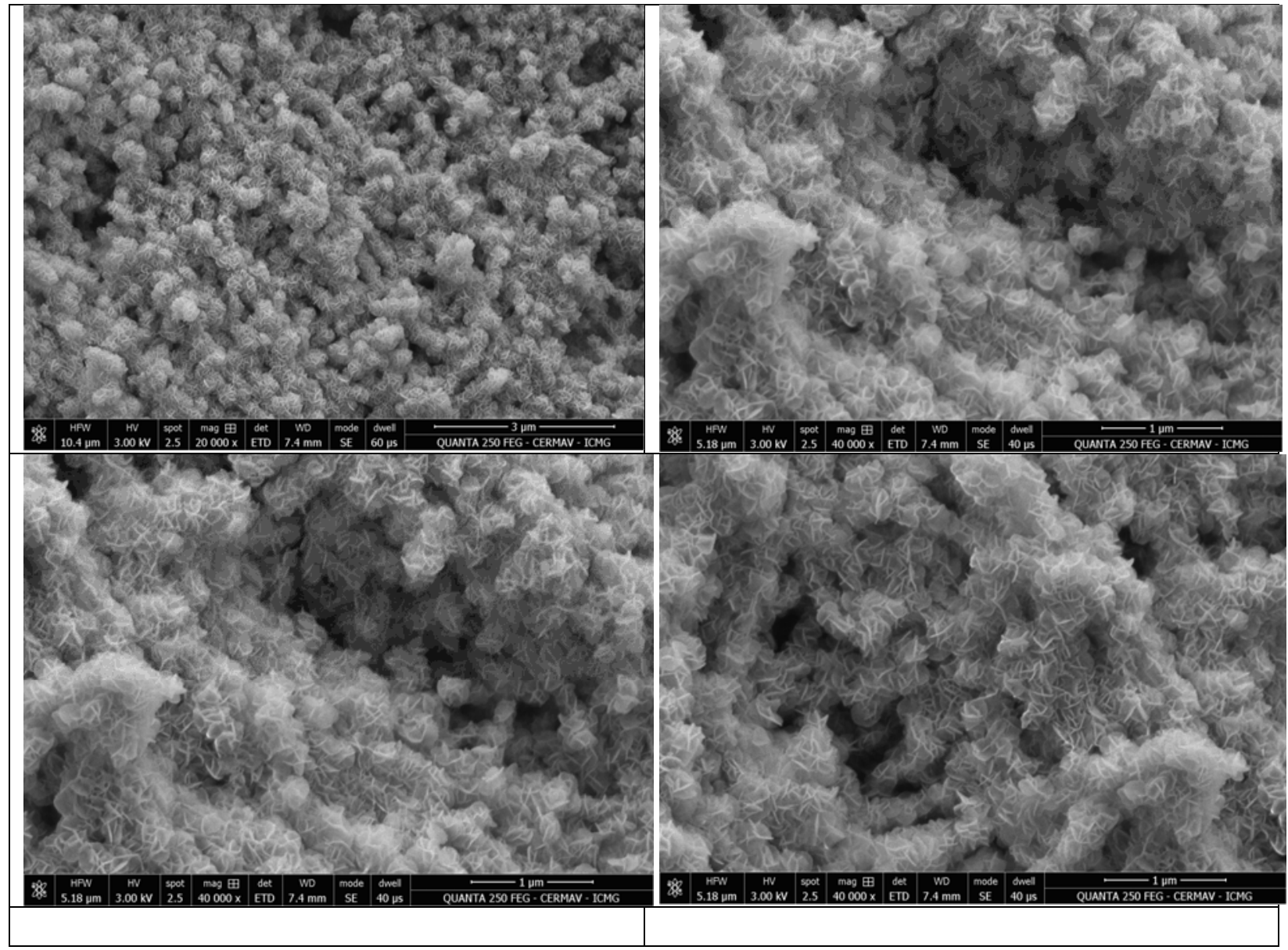

Figure (1): Micrographs of novel nanocoral morphology.

\section{2-Cubosomes and hexosomes}

A systematic exploration of the variation of self-assembled structures of MH1.2k-b-PS2.3k as a function of BCP concentration is shown in Figures $(2,3)$ below. Four different types of particles 
(that is, micelles, lamellae, multilamellar vesicles and cubosomes particles) were observed by transmission electron microscopy (TEM). To which extent each morphology was formed is governed by the final copolymer concentration. The coexistence of different morphologies over rather large range of conditions is often observed in BCP self-assembly in solution caused by the effect of dispersity in block lengths of BCPs. Furthermore, coexistence can be expected if the structure formation is kinetically controlled, which is the case here.

Different types of interactions- including hydrogen bonds, hydrophobic interactions and van der Waals interactions with varying intensities- interplay with each other to create a complex environment for self-assembling and providing multidimensional driven forces for arrangement of molecules to generate cubosomes.

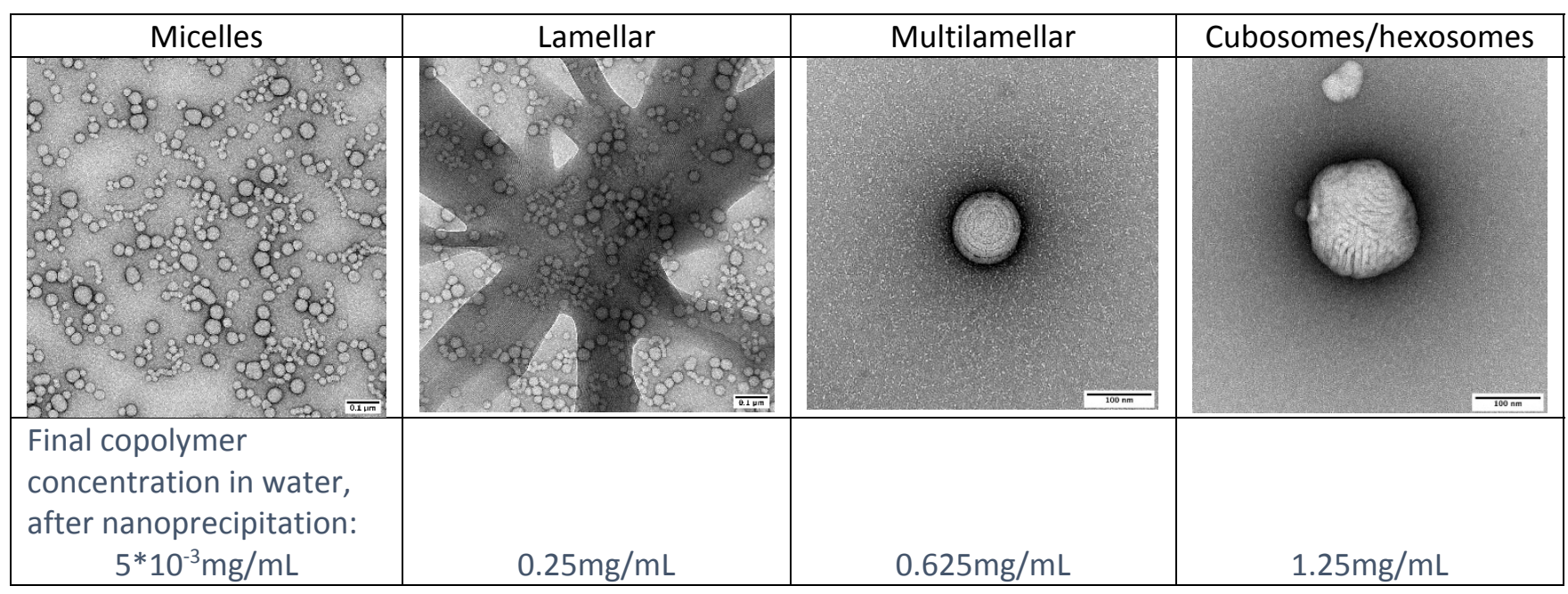

Figure (2): Four different types of particles: micelles, lamellae, multilamellar vesicles and cubosomes particles observed by TEM.

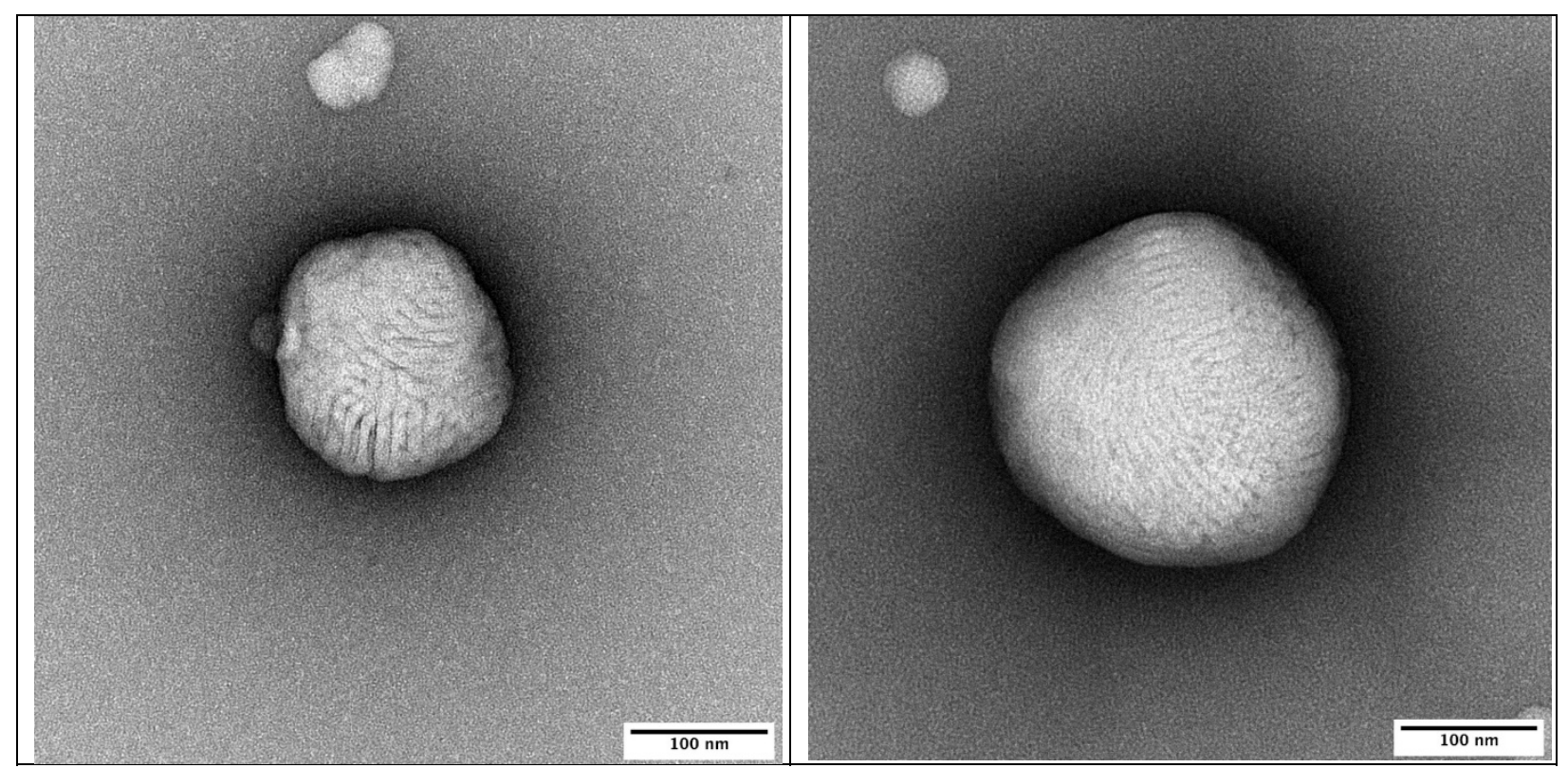




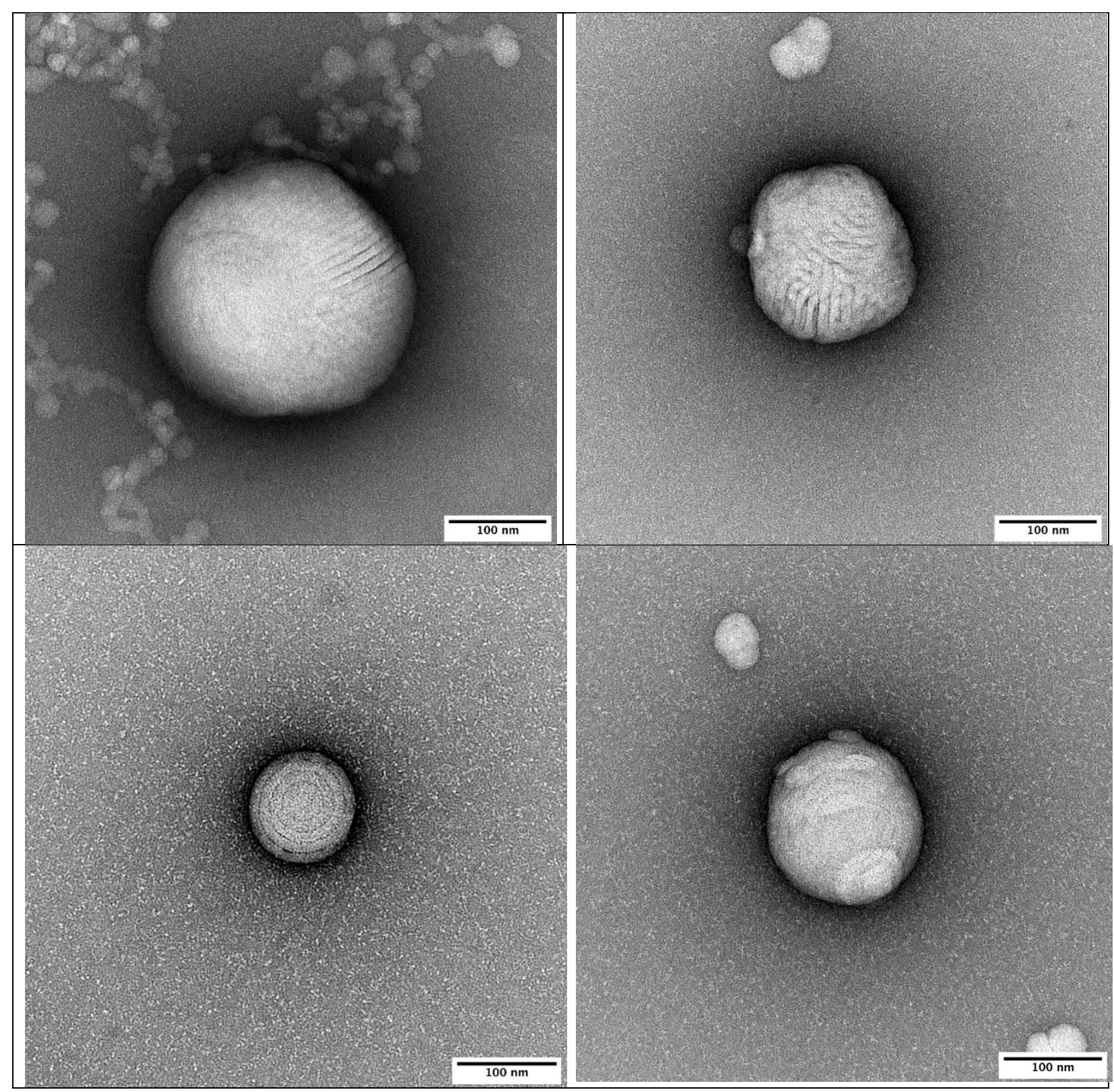

Figure (3): TEM micrographs of novel cubosomes/hexosomes and multi-lamellar morphologies

\section{3-Nanocubes:}

Figure (4) depicts SEM micrographs of nanocubes based on MH1.2k-b-PS2.3k, with molecular faceted crystals. Two key factors were identified for the formation of cubic nanoparticles: (1) the self-assembly of the copolymers in water, and (2) the rate of evaporation.

(1). Role of self-assembly of the copolymers in water:

In a control experiment, homopolymers derived from the two constituent blocks, $\mathrm{MH}$ and PS, were dissolved in water (or acetone) and dried under the same conditions. Neither of these homopolymers formed cubic nanoparticles; while PS formed an amorphous film without any nanostructures, $\mathrm{MH}$ shows a kind of lamellar organization at the solid state, Figures 5 (a,b). 
Furthermore, when the copolymer was dissolved in water/THF 1:2 w/w, which is a good solvent mixture for both blocks, no cubic nanostructures were observed upon drying, Figure 5(c). In sum, the above-mentioned results clearly indicate that the self-assembly of the copolymer in water is essential for the formation of cubes.

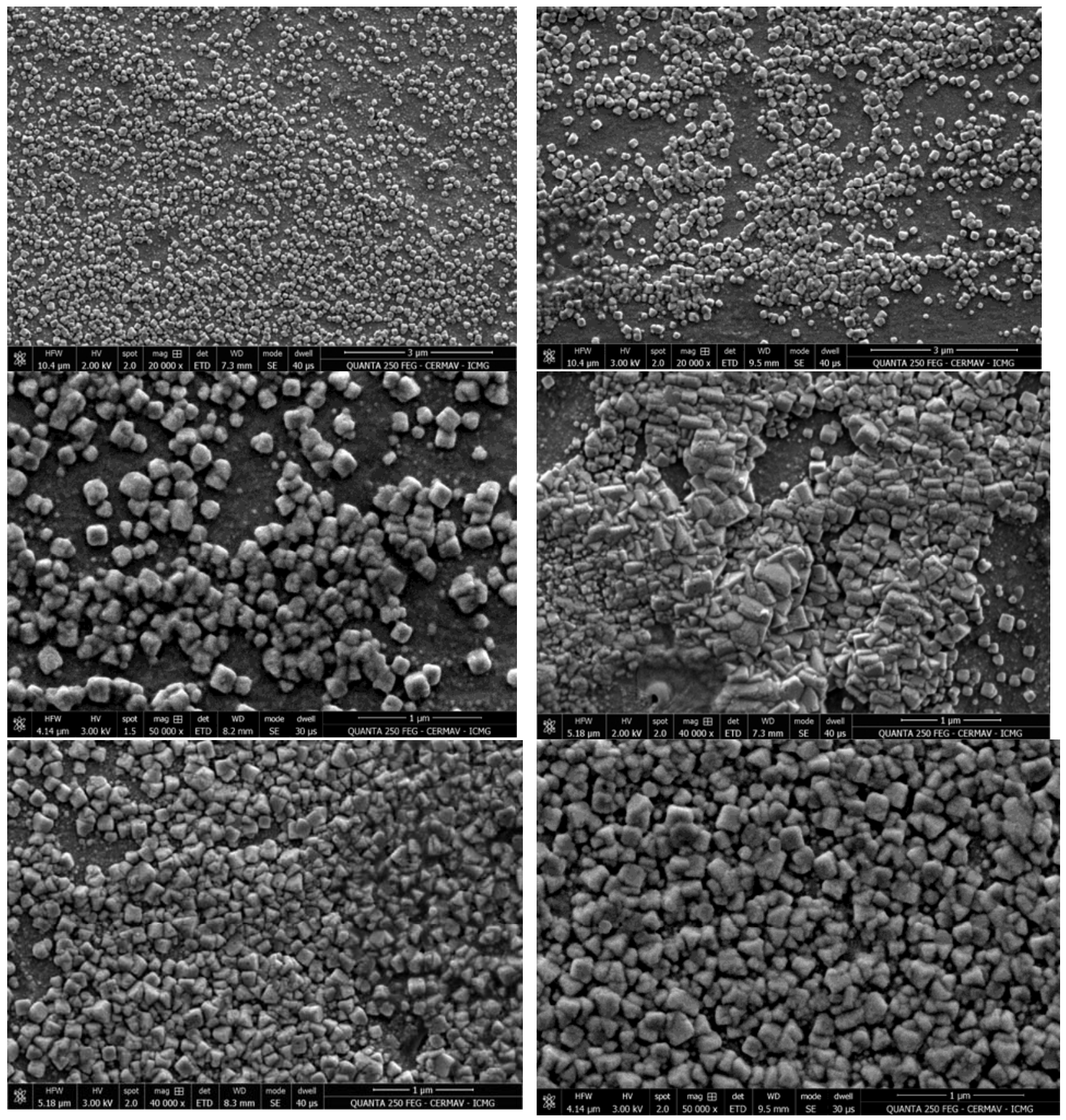

Fig (4): SEM micrographs of nanocubes based on MH1.2k-b-PS2.3k 


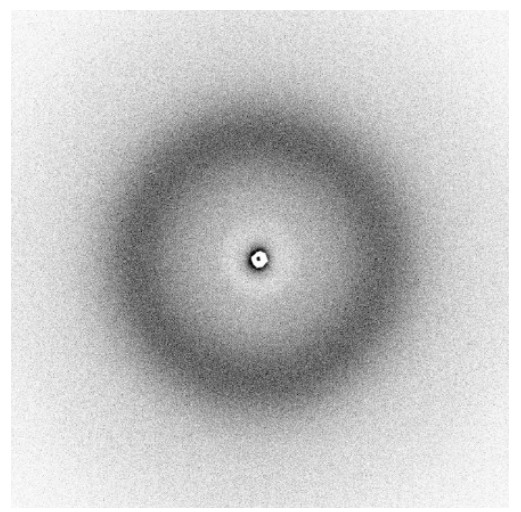

(a)

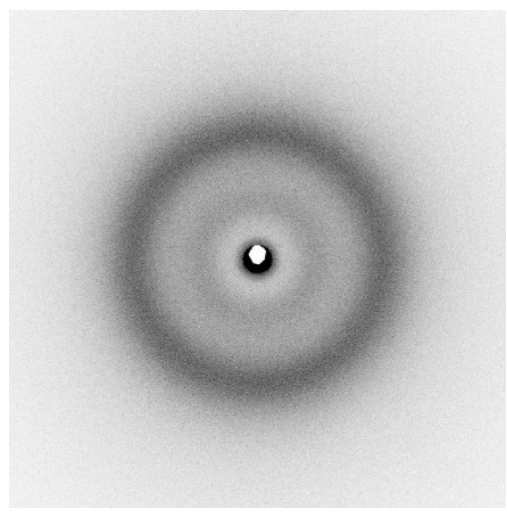

(b)

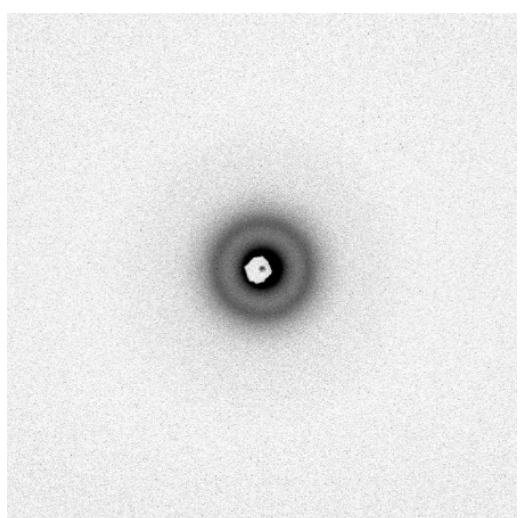

(c)

Fig (5): WAXD patterns of (a) MH, (b) MH1.2k-b-PS2.3k and (c) SAXS patterns of- MH1.2k-bPS2.3k in water/THF 1:2 w/w.

We also examined another copolymer (MH1.2k-b-PS4.8k) with a different hydrophobic block length. Micellar solutions of the second copolymers has undergone the same drying procedure and formed morphologically similar cubic nanoparticles with size ranging from $50 \mathrm{~nm}$ to $90 \mathrm{~nm}$ (Figure 6). Therefore, the formation of cubes is not unique to a specific MH1.2k-b-PS2.3k but is a general phenomenon for this class of diblock copolymers as they are subjected to similar selfassembly in water.

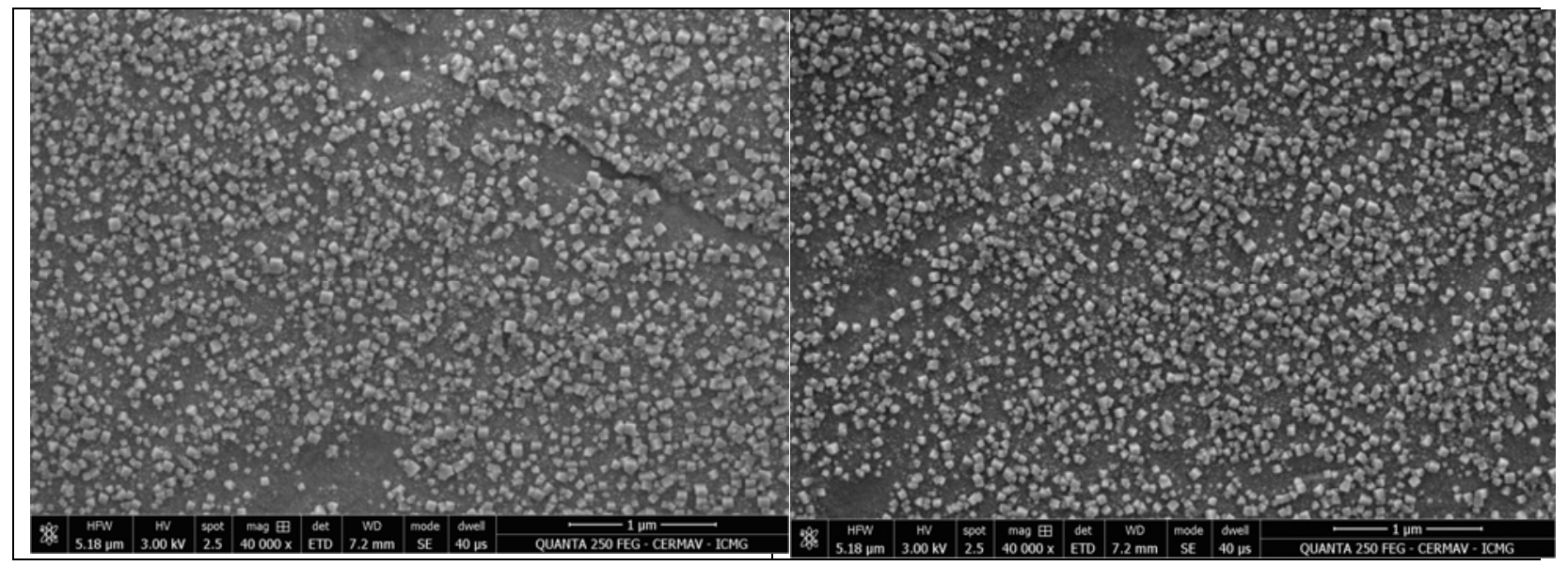




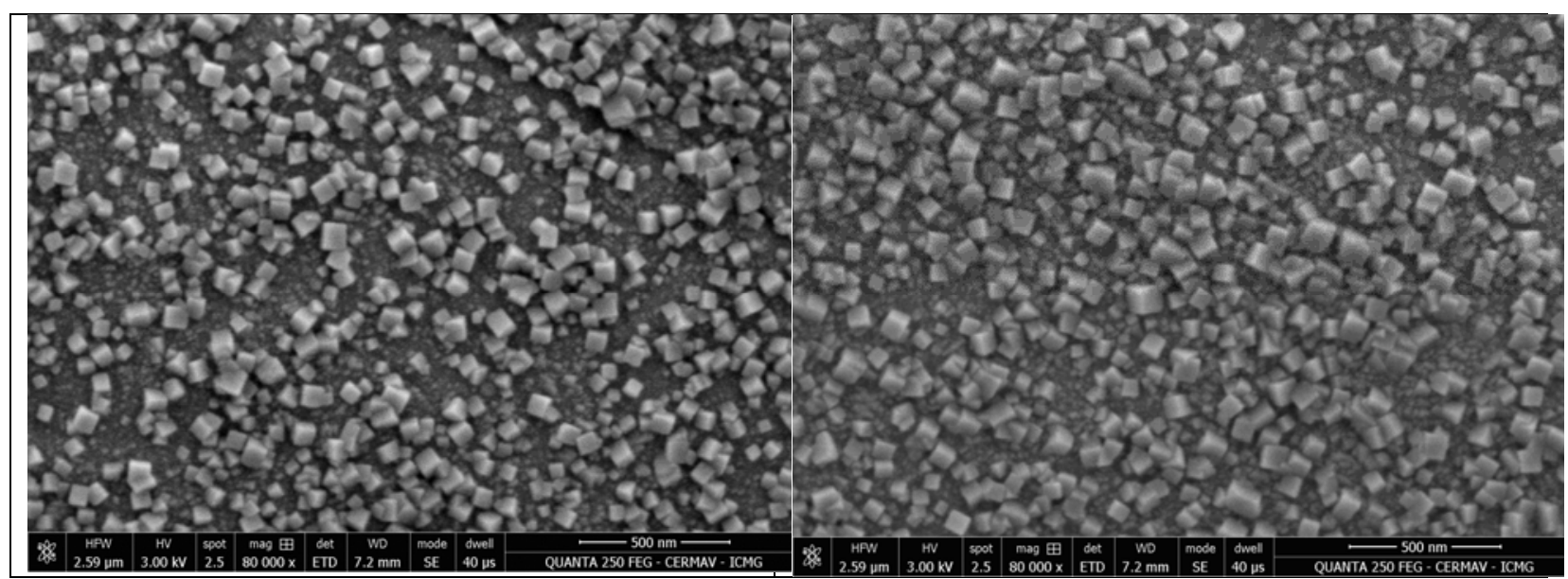

Figure (6): SEM micrographs of nanocubes based on MH1.2k-b-PS4.8k

(2). Role of the rate of evaporation:

Water evaporation rate plays an important role in cube formation as claearly seen in Figure 7. While rapid drying results in an interconnected macroporous network, Figure 7(a), an incomplete drying after $12 \mathrm{~h}$, yields irregularly shaped particles with softer edges, which is suggestive of a transition phase between micellar aggregates and the cubes, Figure 7 (b).

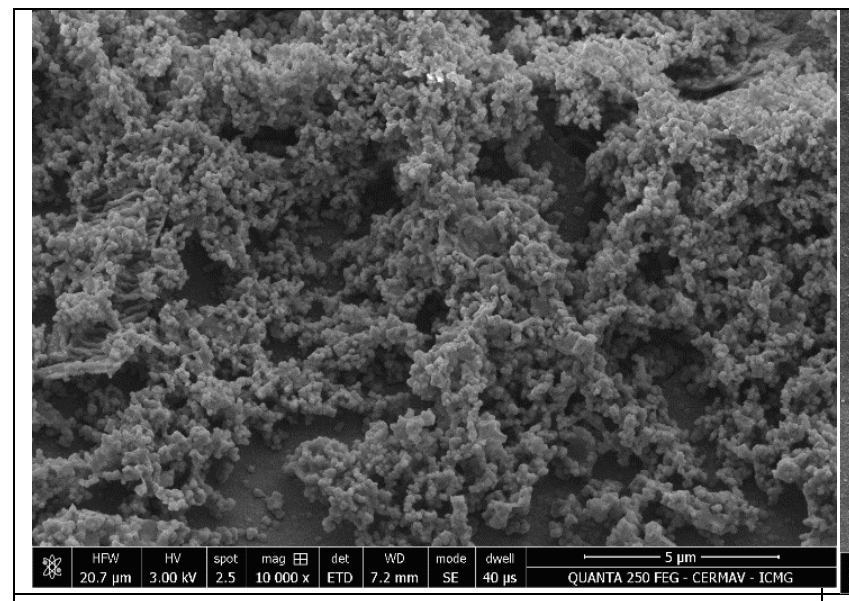

(a)

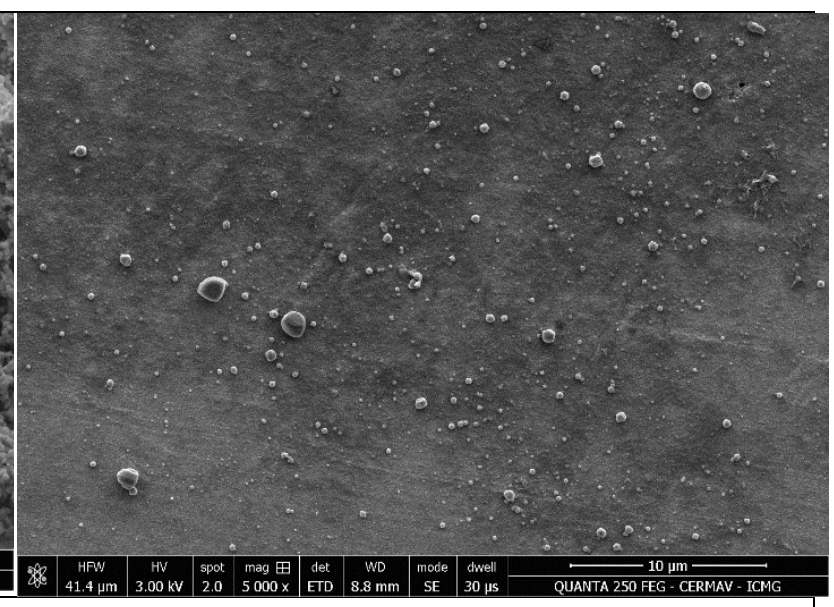

(b)

Figure (7). Effect of drying on the final morphology of the nanostructures, (a) rapid drying at room temperature, and (b) incomplete drying for $12 \mathrm{~h}$.

The cubic nanoparticles assembled from the diblock copolymer micelles readily dissociate to micelles once redispersed in water, as evidenced by SEM micrograph, and DLS, Figures (8-10). The results of DLS study are corroborated by the SEM observations. 


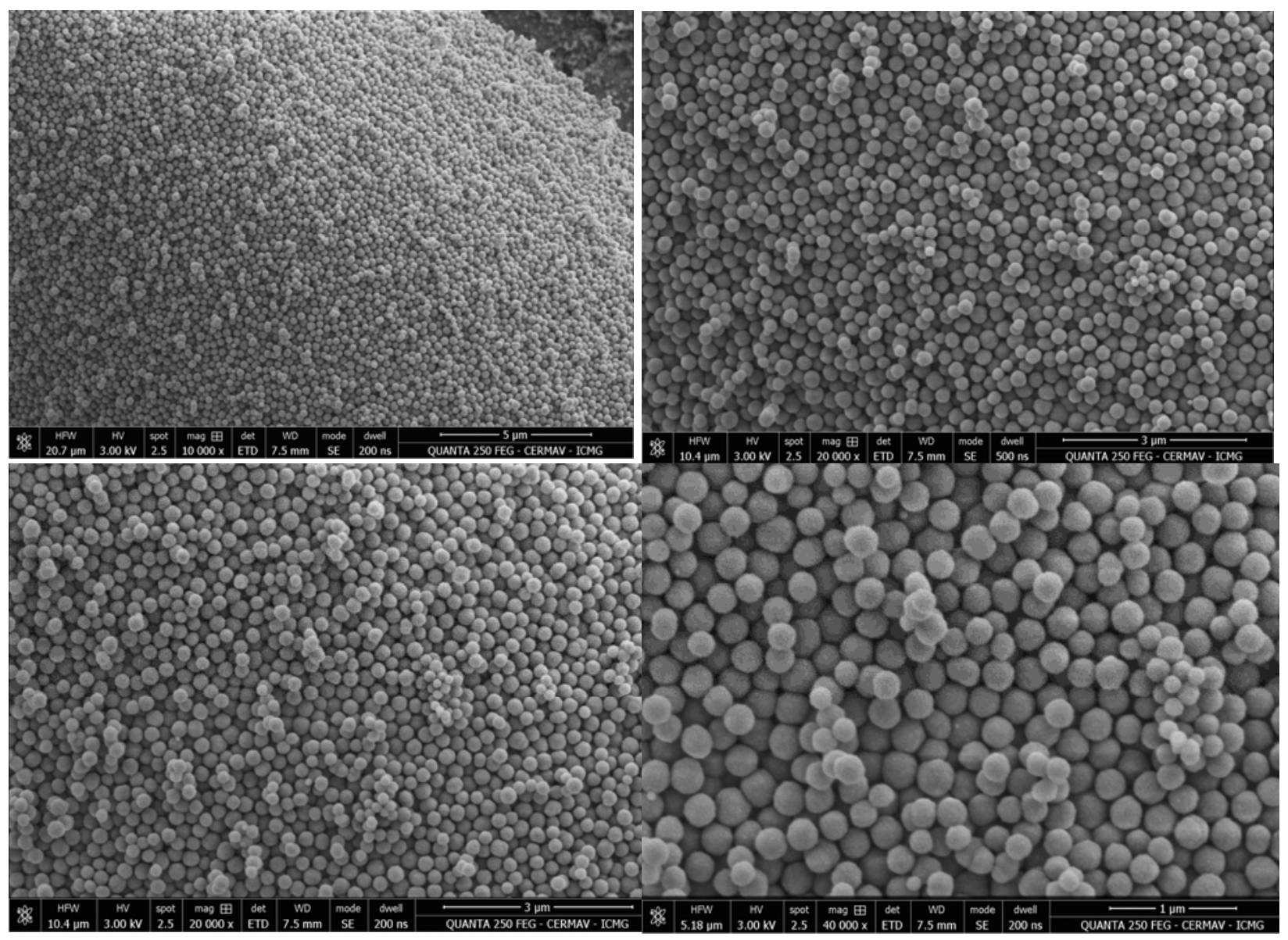

Figure (8): Micrographs of monodispersed nanoparticles in water.

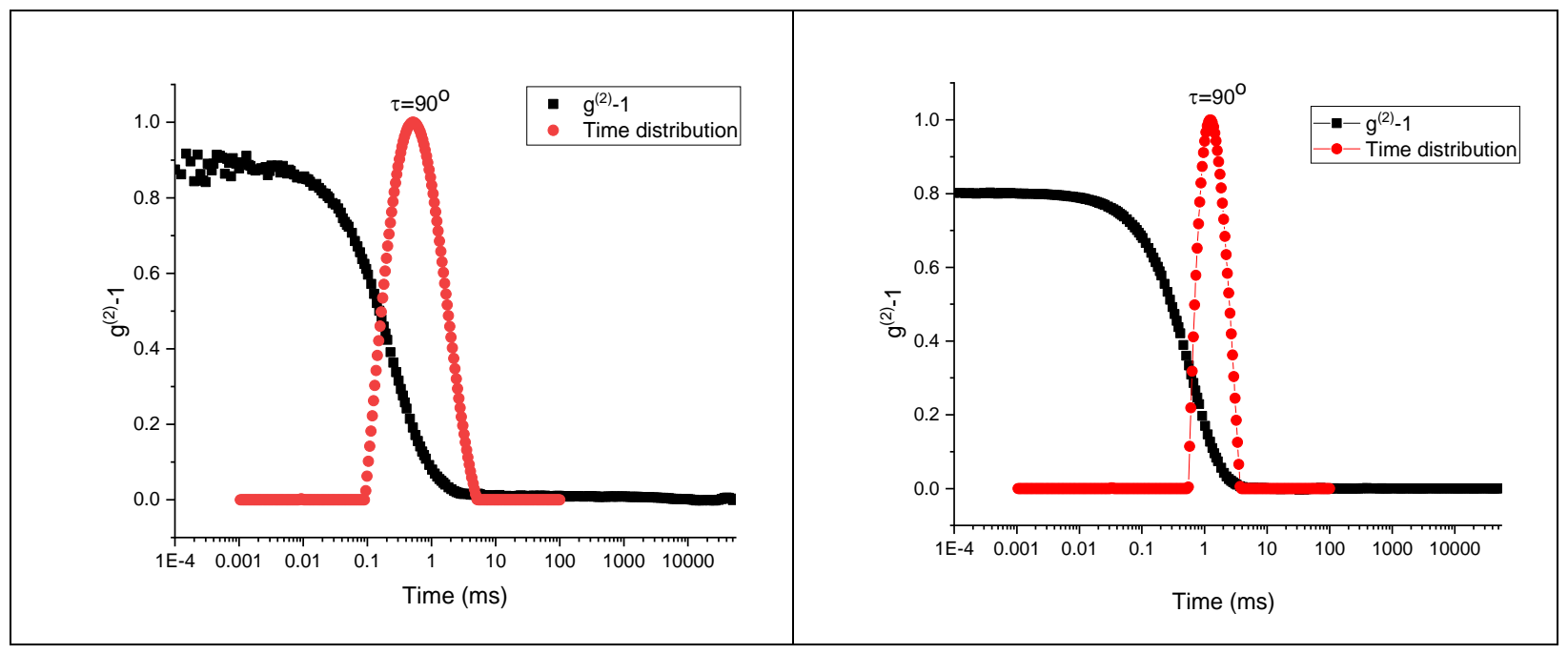


Figure (9): DLS autocorrelation function $\left(\mathrm{g}^{(2-1)}\right.$ measured at $90^{\circ}$ and relaxation-time distribution of the aqueous suspensions of $\mathrm{MH} 1.2 \mathrm{k}-\mathrm{b}-\mathrm{PS} 2.3 \mathrm{k}$ obtained by (A) method A and (B) method B.

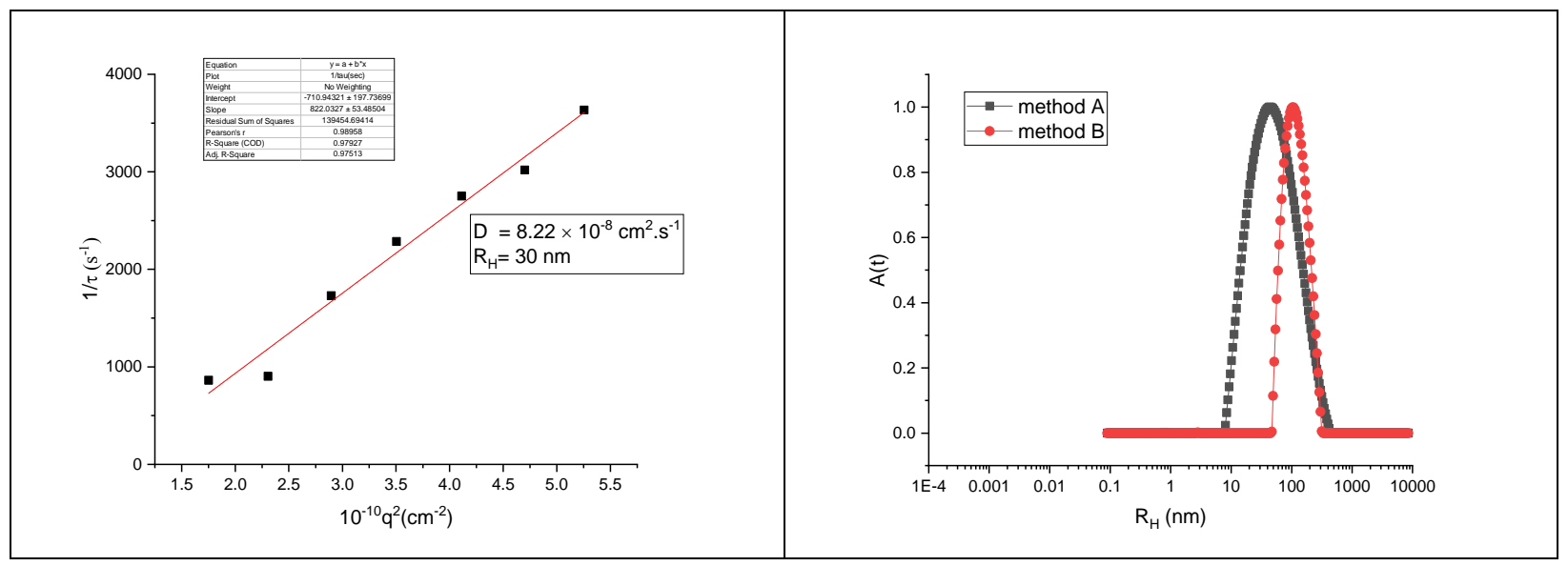

Figure (10): (a) Dependence of the relaxation frequency $(1 / \tau)$ on the squared wave vector modulus (q2) of the aqueous suspensions of MH1.2k-b-PS2.3k obtained by method A, (b) Comparison of the hydrodynamic radius and distribution width between the two methods A and B.

\section{Conclusion}

Polymer self-assembly in solution still constitutes a simple methodology for the preparation of elegant yet sophisticated nanomaterials. This paper has presented how the fine tuning of the experimental parameters of the nanoprecipitation process could lead to a variety of novel morphologies ranging from nanocorals through cubosomes to nanocubes. A carbohydrate dibloc copolymer with a simple and linear structure MH1.2k-b-PS2.3 has been used as a model to illustrate the formation of these new self-assemblies.

We believe that the understanding of the mechanism of formation of these different morphologies could be significantly improved by further studies to selectively favor the formation of a specific self-assembly. This myriad of tailored self-assemblies based on a carbohydrate dibloc copolymer has powerful applicability to various fields, such as cosmetics, pharmaceutics, medicine and so on.

\section{Acknowledgement}

This research was carried out during the visit of Yomen Atassi (HIAST, Damascus, Syria) to CERMAV invited by Nanosciences Foundation. Yomen Atassi would like to thank Alain Fontaine, director of the foundation, for his continuous encouragement and support all the long of the project.

\section{References:}

[1] Z. Jin, H. Fan, Soft Matter, 10, 9212 (2014). DOI 10.1039/C4SM02064B 
[2] D.B. Wright, J.P. Patterson, A. Pitto-Barry, A. Lu, N. Kirby, N.C. Gianneschi, C. Chassenieux, O. Colombani, R.K. O’Reilly, Macromolecules, 48, 6516 (2015).

[3] K. Zhu, D. Wang, J. Liu, Nano Res., 2, 29 (2009). DOI 10.1007/s12274-009-9002-2

[4] J. Liu, T. Luo, S. Mouli, F. Meng, B. Sun, M. Lia, J. Liu, Chem. Commun., 46, 472 (2010). DOI 10.1039/B915650J

[5] R. Ferrari, M. Sponchioni, M. Morbidellia, D. Moscatellib, Nanoscale, 10, 22701 (2018). DOI $10.1039 / \mathrm{C} 8 \mathrm{NR} 05933 \mathrm{~K}$

[6] Z. Karami, M. Hamidi, Drug Discov Today, 21, 789(2016).

[7] H. He, K. Rahimi, M. Zhong, A. Mourran, D.R. Luebke, H.B. Nulwala, M. Moller, K. Matyjaszewski, Nature Comm. 2017, DOI: 10.1038/ncomms14057.

[8] J. F. Alexander, V. Kozlovskaya, J. Chen, T. Kuncewicz, E. Kharlampieva, B. Godin, Adv. Healthcare Mater., 4, 2657 (2015). DOI: 10.1002/adhm.201500537

[9] J. P. Rolland, B. W. Maynor, L. E. Euliss, A. E. Exner, G. M. Denison, J. M. DeSimone, J. Am. Chem. Soc., 127, 10096 (2005). DOI: 10.1021/ja051977c

[10] Y. Mai, A. Eisenberg, Chem. Soc. Rev. 41, 5969 (2012). DOI: 10.1039/c2cs35115c

[11] W. Q. Zhang, L. Q. Shi, Y. L. An, X. D. Shen, Y. Y. Guo, L. C. Gao, Z. Liu, B. L. He, Langmuir, 19, 6026 (2003).

[12] S. Tu, B. L. Wang, Y. W. Chen, Z. M. Li, X. L. Luo, ACS Macro Lett. 1, 933 (2012)

[13] X. Y. Zhang, R. M. Waymouth, J. Am. Chem. Soc., 139, 3822 [2017].

[14] K. Margulis, X. Zhang, L. Joubert, K. Bruening, C.J. Tassone, R.N. Zare, R.M. Waymouth, Angew. Chem. Int. Ed., 56,16357 (2017). DOI: 10.1002/anie.201709564

[15] F. Ganachaud, J. L. Katz, Chem. Phys.Chem., 6, 209 (2005).

[16] E. Lepeltier, C Bourgaux, P Couvreur, Adv. Drug Deliv. Rev., 71, 86 (2014).

[17] S.A. Vitale, J.L. Katz, Langmuir, 19, 4105 (2003). 